\title{
Comunicação
}

[Communication]

\section{Helmintos oxiuridae parasitos de Iguana iguana (Squamata, Lacertilia, Iguanidae) procedentes do Brasil}

\author{
[Oxiuridae helminths parasites of Iguana iguana (Squamata, Lacertilia, \\ Iguanidae) in Brazil] \\ P. Breves ${ }^{1}$, M. Porto $^{1}$, A. Pissinatti ${ }^{2,3}$, D. Luz $^{3}$, R.C. Menezes ${ }^{4}$ \\ ${ }^{1}$ Centro Universitário IBMR \\ Praia de Botafogo, 158 \\ Botafogo- 22250-040 - Rio de Janeiro, RJ \\ ${ }^{2}$ Centro de Primatologia - Rio de Janeiro - Instituto Estadual do Ambiente - Guapimirim, RJ \\ ${ }^{3}$ Centro Universitário Plínio Leite - Itaboraí, RJ \\ ${ }^{4}$ Laboratório de Pesquisa Clínica em Dermatozoonozes - Animais Domésticos - Instituto de Pesquisa Clínica Evandro \\ Chagas - Fundação Oswaldo Cruz - Rio de Janeiro, RJ
}

O lacertídeo Iguana iguana (Linnaeus, 1758) ocorre do México ao sul do Trópico de Capricórnio no Paraguai, e foi introduzido no sul da Flórida e no Havaí (Conant e Collins, 1998). No Brasil, a espécie distribui-se pela região Amazônica, parte da região Centro-oeste, Pantanal e na Caatinga (Ávila-Pires, 1995).

O parasitismo gastrointestinal é bem frequente na clínica veterinária reptiliana, embora a presença de sinais clínicos e a mortalidade dependam do estado de saúde do animal, da espécie e da quantidade de parasitos (Mader, 2006; Loukopoulus et al., 2007). Entretanto, no que diz respeito ao I. iguana, há uma carência de estudos sobre sua fauna helmintológica. Os poucos trabalhos publicados sobre helmintos parasitos desse hospedeiro, tanto de vida livre como de cativeiro, relatam comumente a presença de oxiurídeos, cuja intensidade de infecção é geralmente alta e pode chegar a 5.244 espécimes (Pereira, 1935; Petter, 1969; Werther et al., 2000; Lopes e Silva, 2007).

No Brasil, as espécies de nematoides oxiurídeos relatadas no réptil I. iguana foram: Ozolaimus magatyphlon (Rudolphi, 1819) Dujardin, 1845, Ozolaimus cirratus Linstow, 1906, Alaeuris vogelsangi (Lent e Freitas, 1948) Peter e Quentin, 1976 e Alaeuris caudatus (Lent e
Freitas, 1948) Peter e Quentin, 1976, de ocorrência em animais de vida livre nos estados do Ceará (Lopes e Silva, 2007), Pernambuco (Petter, 1969) e Paraíba (Pereira, 1935) e em um iguana de cativeiro no estado de São Paulo (Werther et al., 2000). O sítio de infecção encontrado para os nematoides $O$ magatyphlon, $O$. cirratus e A. caudatus foi o intestino grosso, e para o helminto $A$. vogelsangi o esôfago, o estômago e o intestino grosso (Pereira, 1935; Petter, 1969; Werther et al., 2000; Lopes e Silva, 2007).

Neste trabalho são apresentados os resultados de estudo sobre a fauna de nematoides gastrointestinais ocorrentes em I. iguana de vida livre proveniente do Nordeste, Alagoas e Maranhão, e Centro-oeste, Goiás e Mato Grosso, do Brasil. Foram necropsiados no ano de 2008 seis iguanas adultos, três machos e três fêmeas, de vida livre, oriundos dos estados do Maranhão, Alagoas, Mato Grosso e Goiás. Estes animais encontravam-se depositados na coleção do Museu Nacional da Universidade Federal do Rio de Janeiro, conservados em álcool a $70^{\circ} \mathrm{GL}$, sob os números 1796, 3914, 5014, 6445, 11349, 11350, tendo sido coletados, respectivamente, nos anos de 1908, 1988, 1997, 1986, 2004 e 2004.

Recebido em 7 de novembro de 2010 
$\mathrm{Na}$ necropsia, os órgãos do sistema digestório foram retirados e abertos com auxílio de uma tesoura em placas de Petri separadas. As regiões do canal alimentar examinadas, identificadas de acordo com Parsons e Cameron (1977), foram: esôfago, estômago e intestinos delgado e grosso. Os helmintos encontrados foram processados segundo as técnicas helmintológicas descritas por Pessoa e Martins (1988), identificados de acordo com Pereira (1935) e Vicente et al. (1993) e depositados no Laboratório de Ecologia do Departamento de Endemias, da Escola Nacional de Saúde Pública - FIOCRUZ, sob os números: MNRJ 1796,3914,5014,6445,11349 e 11350. As fotomicrocrafias foram realizadas com a câmera LEICA DFC 420. As medidas são apresentadas em milímetros. A intensidade de infecção foi considerada alta quando acima de 100 espécimes de helmintos.

Todos os seis lacertídeos I. iguana apresentavam alta intensidade de infecção por nematoides. Os helmintos encontrados foram os nematoides oxiurídeos: Ozolaimus cirratus (Fig. 1A, 1D) no ceco e cólon de cinco iguanas; Ozolaimus megatyphlon (Fig. 1B, 1E) no ceco, cólon e reto de três; e Alaeuris vogelsangi (Fig. 1C, 1F) no intestino delgado, ceco, cólon e reto de cinco animais. Duas larvas de Ozolaimus sp. foram coligidas na região pilórica do estômago de um iguana. Os iguanas encontravam-se parasitados por duas ou três espécies de oxiurídeos, com exceção de um único espécime, que estava infectado somente por $O$. cirratus.

As medidas dos nematoides identificados encontram-se na Tab. 1. Foram observadas tanto fêmeas ovíparas como fêmeas vivíparas de $A$. vogelsangi (Fig.1F). O. cirratus e A. vogelsangi foram encontrados em Alagoas, Goiás, Maranhão e Mato Grosso, e O. megatyphlon só não foi registrado no Maranhão.
Todas as três espécies de oxiurídeos encontradas já haviam sido relatadas no Brasil (Vicente et al., 1993). Entretanto, foi o primeiro registro desses nematoides na região Centro-Oeste e nos estados de Alagoas e Maranhão.

A ocorrência de fêmeas vivíparas e ovíparas em A. vogelsangi somente havia sido relatada por Petter (1969). Segundo esse autor, essa poecilogonia ocorre em oxiurídeos parasitos de répteis herbívoros de regiões caracterizadas por uma estação seca e, provavelmente, representa uma adaptação à ecologia do hospedeiro.

Pereira (1935), Lent e Freitas (1948), Petter (1969) e Werther et al. (2000) relataram que o intestino grosso é o único sítio de infecção do gênero Ozolaimus. Todavia, no presente estudo, e no trabalho de Munakata et al. (1999), esse helminto foi encontrado no estômago. A. vogelsangi foi a única espécie encontrada no intestino delgado. Esse sítio de infecção já havia sido reportado para essa espécie por Lopes e Silva (2007). Entretanto, no presente artigo, o sítio de infecção mais comum de $A$. vogelsangi foi o intestino grosso e não o estômago e o esôfago, como relatado por esses autores.

A alta frequência e a intensidade de infecção encontradas para as três espécies de oxiurídeos foram semelhantes às relatadas em outros trabalhos, tanto em iguanas de vida livre como em iguanas de cativeiro (Munataka et al., 1999; Lopes e Silva, 2007; Loukopoulos et al., 2007). $\mathrm{O}$ fato de serem helmintos muito comuns e de causarem mortalidade em animais criados em cativeiro (Munakata et al., 1999; Loukopoulus et al. 2007) torna imprescindível a inclusão desses oxiurídeos dentro do programa de monitoramento sanitário desses animais. 

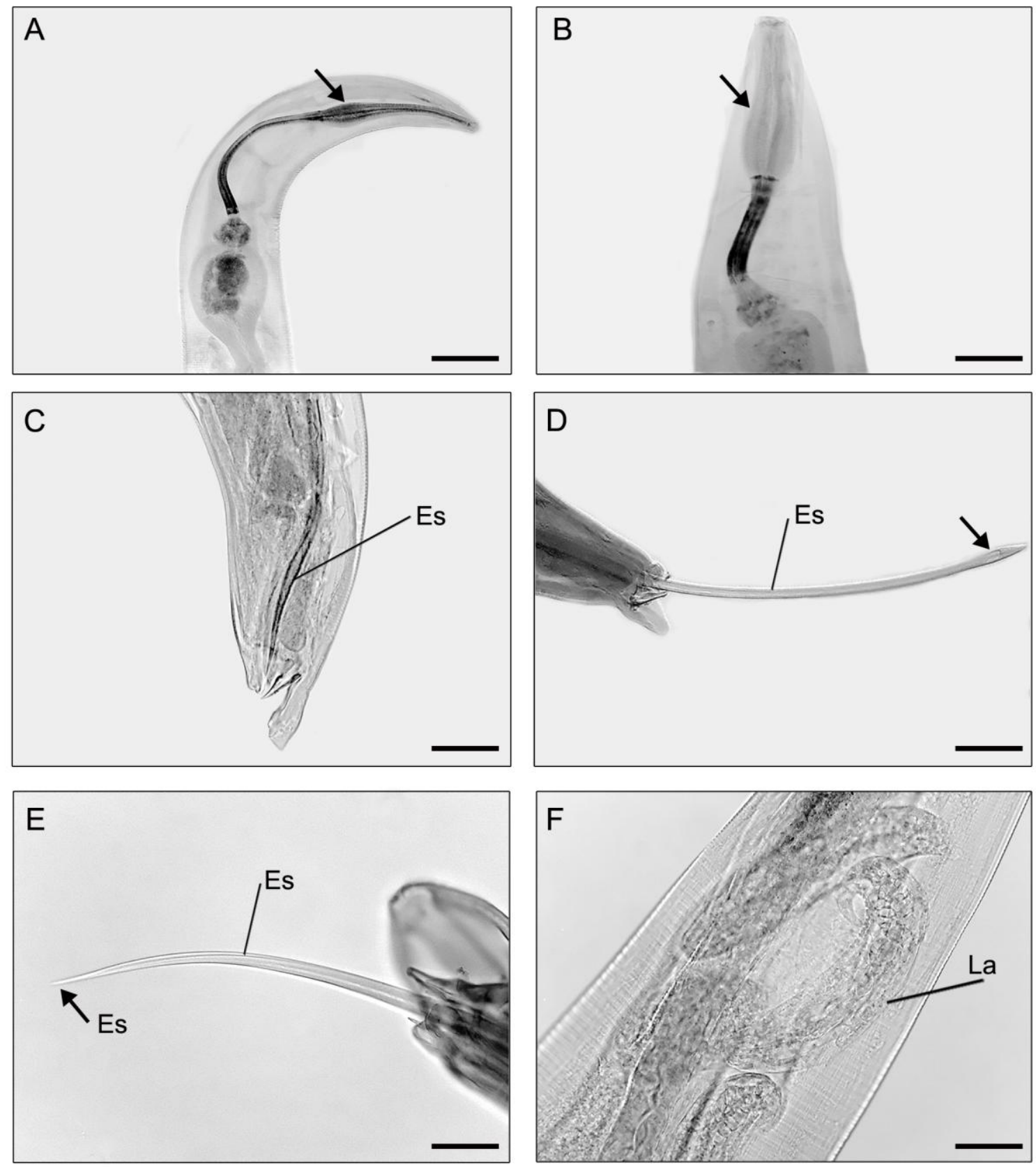

Figura 1. Nematoides encontrados em iguanas de vida livre. A) Região anterior de Ozolaimus cirratus mostrando dilatação (seta) na porção central do esôfago. Barra=0,3mm. B) Região anterior de Ozolaimus megatyphlon mostrando dilatação (seta) em toda a região anterior do esôfago. Barra= $0,4 \mathrm{~mm}$. C) Região posterior de macho de Alaeuris vogelsangi mostrando espículo (Es). Barra=0,04mm. D) Extremidade posterior de macho de Ozolaimus cirratus mostrando espículo (Es) com dilatação (seta) anterior a sua ponta. Barra $=0,16 \mathrm{~mm}$. E) Extermidade posterior de macho de O. megatyphlon mostrando espículo (Es) terminado em ponta fina (seta). Barra=0,04mm. F) Região posterior de fêmea de Alaeuris vogelsangi mostrando larvas (La) no útero. Barra $=0,04 \mathrm{~mm}$. 
Helmintos oxiuridae parasitos...

Tabela 1. Medidas (mm) de Ozolaimus cirratus, Ozolaimus megatyphlon e Alaeuris vogelsangi encontrados em iguanas de vida livre provenientes das regiões Nordeste e Centro-oeste do Brasil e depositados no Museu Nacional da Universidade Federal do Rio de Janeiro

\begin{tabular}{|c|c|c|c|c|c|c|}
\hline \multirow{2}{*}{ Medidas } & \multicolumn{2}{|c|}{ O. cirratus } & \multicolumn{2}{|c|}{ O. megatyphlon } & \multicolumn{2}{|c|}{ A. vogelsangi } \\
\hline & Macho & Fêmea & Macho & Fêmea & Macho & Fêmea \\
\hline Comprimento & $4,9-6,54$ & $6,38-8,73$ & $3,67-4,76$ & $4,99-6,97$ & $2,27-2,7$ & $3,42-3,94$ \\
\hline Largura & $0,57-0,6$ & $0,84-1,1$ & $0,34-0,4$ & $0,56-0,85$ & $0,2-0,24$ & $0,25-0,31$ \\
\hline $1^{\mathrm{a}}$ porção do esôfago & $0,74-0,91$ & $1,05-1,16$ & $0,66-0,96$ & $1,04-1,16$ & A & A \\
\hline $2^{-\mathrm{a}}$ porção do esôfago & $0,89-1,13$ & $1,19-1,36$ & $0,89-1,22$ & $1,39-1,71$ & A & A \\
\hline Esôfago total & $1,65-2,04$ & $2,25-2,45$ & $1,68-2,09$ & $2,43-2,87$ & $0,66-1,08$ & $0,78-1,29$ \\
\hline Bulbo esofagiano & $0,20-0,27 x$ & $0,29-0,37 x$ & $0,10-0,19 x$ & $0,18-0,25 x$ & $0,08-0,15 \mathrm{x}$ & $0,11-0,18 x$ \\
\hline & $0,21-0,25$ & $0,27-0,34$ & $0,16-0,20$ & $0,18-0,28$ & $0,09-0,16$ & $0,10-0,20$ \\
\hline $\begin{array}{c}\text { Anel nervoso a } \\
\text { extremidade anterior }\end{array}$ & $0,2-0,3$ & $0,4-0,6$ & $0,2-0,3$ & $0,3-0,8$ & 0,1 & $0,1-0,2$ \\
\hline Espículo & $2,08-2,37$ & A & $0,72-1,03$ & A & $0,16-0,19$ & A \\
\hline Vulva a cauda & A & $1,4-3,03$ & A & $0,98-1,58$ & A & $1,62-1,76$ \\
\hline Ovos & A & $\begin{array}{c}0,12-0,14 x \\
0,06-0,10\end{array}$ & A & $\begin{array}{c}0,10-0,15 \times \\
0,06-0,09\end{array}$ & A & $\begin{array}{c}0,20-0,28 \times \\
0,14-0,16\end{array}$ \\
\hline
\end{tabular}

$\mathrm{A}=$ ausente.

Palavras-chave: Iguana iguana, parasitos, sistema digestivo, nematoides, réptil

\section{ABSTRACT}

The results of a study on nematode fauna occurring in wild iguanas (Iguana iguana) from Brazilian Northeast (Alagoas and Maranhão) and Central-west (Goiás and Mato Grosso) areas were presented. Six adult iguanas, three males and three females, were necropsied and the digestive system removed to examination. All the iguana specimens were heavily parasitized. The helminths diagnosed were: Ozolaimus cirratus in the cecum and colon of five iguanas; Ozolaimus megatyphlon in cecum, colon and rectum of three iguanas; and Alaeuris vogelsangi in the small intestine, cecum, colon and rectum of five animals. Two larvae of Ozolaimus sp. were recovered from the pyloric region of the stomach of one iguana. The three diagnosed species of nematodes were reported for the first time in the Brazilian Central-West region.

Keywords: Iguana iguana, digestory sistem, nematodes, reptiles

\section{AGRADECIMENTOS}

Ao Dr. Ronaldo Fernandes, do setor de Herpetologia do Museu Nacional do Rio de Janeiro - UFRJ, pelo empréstimo dos iguanas. Ao Rodrigo Méxas, Laboratório de Imagens, Instituto Oswaldo Cruz, pela assistência técnica relativa às figuras. À Fundação de Amparo a Pesquisa do Estado do Rio de Janeiro (FAPERJ) (Proc. E- 26/171.271/2006).

\section{REFERÊNCIAS BIBLIOGRÁFICAS}

AVILA-PIRES, T. C. Lizards of Brazilian Amazonia (Reptilia Squamata). Zool. Verh., v.299, p. 1-706, 1995.

CONANT, R.; COLLINS, J.T. A Field Guide: Reptiles and amphibians of eastern \& central North America. 3.ed. New York: Houghton Mifflin, 1998. 634p.
LENT, H.; FREITAS, J.F.T. Uma coleção de nematoides parasitos de vertebrados do Museu de História Natural de Montevidéo. Mem. Inst. Oswaldo Cruz, v.46, p.1-71, 1948.

LOPES, S.G.; SILVA, L.E.M. Infecção por helmintos em três espécies de lagartos do Nordeste brasileiro. Cad. Cult. Cienc., v.1, p.4751, 2007.

LOUKOPOULOS, P.; KOMNENOU, A.; PAPADOPOULOS, E. et al. Lethal Ozolaimus megatyphlon infection in a green iguana (Iguana iguana rhinolopa). J. Zoo Wildl. Med., v.38, p.131-134, 2007.

MADER, D.R. Reptile medicine and surgery. Philadelphia: W.B. Saunders, 2006. 1264p.

MUNAKATA, Y.; INOUE, I.; SHIRAI, A. Ozolaimus megatyphlon (Nematoda: Pharyngodonidae) isolated from a Green Iguana (Iguana iguana rhinolopa). J. Jpn. Vet. Med. Assoc., v.52, p.784-787, 1999. 
PARSONS, T.S.; CAMERON, J.E. Internal Relief of the Digestive Tract. In: GANS, C. (Ed). Biology of reptilia. 6.ed. New York: Academic, 1977. p.159-224.

PEREIRA, C. Os Oxyurata parasitos de Lacertilia de Nordeste brasileiro. Arq. Inst. Biol., v.6, p.5-27, 1935.

PESSOA, S.B.; MARTINS, A.V. Parasitologia médica. 11.ed. Rio de Janeiro: Guanabara Koogan, 1988. 872 p.

PETTER, A.J. Deux cas de Poecilogynie chez Les Oxyures Parasites D. Iguana iguana (L.). Bull. Mus. Nat. Hist. Nat. Paris, v.41, p.12521260, 1969.
VICENTE, J.; RODRIGUES, H.O.; GOMES, D.C. et al. Nematoides do Brasil. Parte III: nematoides de répteis. Rev. Bras. Zool., v.10, p.10-168, 1993.

WERTHER, K.; VICENTE, J.J.; PETTA, C.B.

Description of a gastrointestinal parasite found in an Iguana (Iguana iguana) in Brazil. 2000.

Disponível em:

<http://www.vet.uga.edu/vpp/ivcvm/2000/werthe r/index.php>. Acessado em: 26 mai. 2008. 